Received: 21 August 2018

Accepted: 17 July 2019

Published online: 31 July 2019

\section{Prognostic value of serum creatine level in patients with vulvar cancer}

\author{
Richard Schwameis ${ }^{1}{ }^{1}$, Magdalena Post $\left.\right|^{1}$, Christine Bekos ${ }^{1}$, Lukas Hefler ${ }^{2,3}$, \\ Alexander Reinthaller ${ }^{1,4}$, Veronika Seebacher ${ }^{1}$, Christoph Grimm $\mathbb{D}^{1}$, Stephan Polterauer ${ }^{1,4}$ \& \\ Samir Helmy-Bader ${ }^{1}$
}

Vulvar cancer is a rare malignancy with poor prognosis that generally occurs in elderly patients. The individual prognosis is difficult to assess. Serum creatinine levels are frequently elevated in elderly patients. Recent evidence have shown shown that - besides indicating kidney impairment - serum creatinine levels may be used to predict the survival in cancer patients. Several studies observed an association between elevated serum creatinine levels and poor prognosis in patients with solid tumors. In this retrospective cohort study, serum creatinine levels were evaluated in 170 patients with invasive vulvar cancer. Serum creatinine levels were correlated to established clinicopathologic factors. Univariate and multivariate survival analysis were performed. Elevated serum creatinine levels ( $>1.2 \mathrm{mg} / \mathrm{dl}$ ) were significantly associated with both poor disease specific and overall survival. Three year overall survival rates were $\mathbf{7 4 . 8 \%}$ and $32.5 \%$ for patients with serum creatinine levels of $\leq$ and $>1.2 \mathrm{mg} / \mathrm{dl}$, respectively. In a multivariate survival model, serum creatinine levels were significantly associated with overall survival independent of tumor stage and patients' age. In conclusion, pretherapeutic serum creatinine levels may be useful as an independent prognostic parameter in patients with vulvar cancer.

Vulvar cancer is a rare gynecological malignancy with a slightly rising incidence rate ${ }^{1,2}$. It is associated with poor survival when compared to other gynecological malignancies. Although dependent on the tumor stage, it is difficult to predict the individual outcome of patients with vulvar cancer, because the survival of patients within the distinct tumor stages may vary significantly ${ }^{3-5}$.

For an individual outcome prediction of patients with vulvar cancer, several factors were considered in the past. On one hand, pathological parameters such as tumor resection margin, lymph node involvement and lymph node ratio were evaluated ${ }^{3,6,7}$. On the other hand, clinical parameters were also shown to be associated with patient survival. Recent studies observed an association between inflammatory biomarkers including C-reactive protein, hypoalbuminemia and fibrinogen to poor survival in patients with vulvar cancer ${ }^{8,9}$.

Interestingly, in a variety of malignancies including patients with sarcoma and urothelial carcinoma, renal impairment delineated by high serum creatinine levels has been associated with poor survival ${ }^{10,11}$. The underlying mechanism for this association is poorly understood ${ }^{12}$.

Impaired renal function is more frequent in patients with $>65$ years than in younger patients ${ }^{13}$. Although the age of patients developing vulvar cancer has slightly decreased within the last decades, the median age at diagnosis still remains at 68 years ${ }^{1}$. Thus it seems reasonable to assess whether renal impairment reflected by elevated kidney function parameters have a prognostic value in patients suffering from vulvar cancer.

Hence this study was performed to assess whether pretherapeutic serum creatinine levels have a prognostic value in patients with invasive vulvar cancer and whether it might serve as a cheap, readily available prognostic parameter.

\footnotetext{
${ }^{1}$ Gynecologic Cancer Unit, Comprehensive Cancer Center, Medical University of Vienna, Waehringer Guertel 18-20, 1090, Vienna, Austria. ${ }^{2}$ Department of Gynecology, Ordensklinikum Linz, Seilerstätte 4, 4020, Linz, Austria. ${ }^{3}$ Karl Landsteiner Institute for Gynecologic Surgery and Oncology, Seilerstaette 4, 4020, Linz, Austria. ${ }^{4}$ Karl Landsteiner Institute for General Gynecology and Experimental Gynecologic Oncology, Waehringer Guertel 18-20, 1090, Vienna, Austria. Correspondence and requests for materials should be addressed to C.G. (email: christoph.grimm@ meduniwien.ac.at)
} 


\begin{abstract}
Materials and Methods
Patients. This study included a total of 341 consecutive patients diagnosed with vulvar cancer that were treated between 1993 and 2016 at the Comprehensive Cancer Center Vienna, Vienna. Patients' clinical data was harvested from available tumor databases and by review of electronic charts. Tumor staging was performed according to the 2009 International Federation of Gynecology and Obstetrics (FIGO) classification system ${ }^{14}$.

Diagnosis of vulvar cancer was established histologically by biopsy. Tumor assessment was performed by clinical examination, and if advanced tumor stage was assumed magnetic resonance imaging (MRI) and computed tomography (CT) were performed.

Patients were treated according to local standards and international guidelines. In early stages of the disease treatment included radical surgery with or without lymph node assessment. Clinically indicated patients received either adjuvant radiotherapy or in individual cases chemotherapy. In advanced stage disease palliative radiotherapy as well as chemotherapy with and without salvage surgery were performed. Table S1 of the supplemental material shows the treatment of patients in detail. All patients were included into the institutions follow up program that included clinical examination and imaging studies if necessary for a total of 10 years. All patients were seen four times annually for three years, twice per year in the following three years and, once annually for further four years. If recurrence was assumed biopsy was taken and imaging studies performed if required.

Approximately 24 to 72 hours prior to treatment patients were subjected to physical examination by a specialist for internal medicine. The examination included evaluation of medical history, a physical examination including blood pressure measurement, auscultation of the lungs, and evaluation of blood values including serum electrolytes, coagulation parameters, blood cell count liver function and renal function tests. If necessary additional examinations for example ECG, chest x-ray or pulmonary function tests were performed.

Patients with FIGO stage Ia and previously known chronic renal disease and patients where necessary values were missing were excluded from analysis.
\end{abstract}

Ethics. Prior to initiation, this study was approved by the Institutional Review Board of the Medical University of Vienna (IRB approval number: 1901/2017). All patients gave consent to treatment according to institutional guidelines and to anonymized assessment of clinical data and treatment outcome. This was a retrospective trial. Therefore, the Institutional Review Board of the Medical University of Vienna waived the requirement to obtain distinct written informed consent from the patients.

Patient data were anonymized and de-identified prior to analysis. The study was performed according to the declaration of Helsinki, the ICH Harmonized Tripartite Guideline for Good Clinical Practice and the guidelines of the Institutional Review Board of the Medical University of Vienna.

Creatinine measurements. As a part of the physical examination prior to treatment serum creatinine levels were assessed. The Creatinine Jaffé Gen. 2 test (COBAS CREJ2; Roche Diagnostics, Indianapolis, Indiana, USA) was used for serum creatinine level measurements and serum creatinine concentrations between 0.50 and $1.2 \mathrm{mg} / \mathrm{dl}$ were considered normal.

Statistical analysis. All data is given as mean ( \pm SD). Students' $t-$ test and one-way Anova were used to compare mean serum creatinine levels and clinic-pathologic variables, where appropriate. The product-limit method of Kaplan and Meier was used to calculate survival probabilities. Differences between groups were assessed by log-ranks test. Univariate and multivariate survival analysis were performed including patients' age ( $\leq$ vs. $>67.7$ years) FIGO tumor stage (FIGO I vs. II vs. III vs. IV), histological grade (G1 vs. G2-G3), lymph node involvement (no involvement [N0] vs. involvement [N1]) and serum creatinine level. The results were analyzed for the endpoint of disease specific and overall survival, with overall survival being defined the primary endpoint. Overall survival was defined as time from the date of diagnosis to the date of death or date of last observation. Survivors were censored on the last date they were known to be alive. Disease specific survival was defined as time from date of diagnosis until date of death due to vulvar cancer (or sequels of vulvar cancer). Patients that died from causes other than vulvar cancer, and survivors were censored at the last date they were known to be alive. Multivariate cox regression models were established to assess serum creatinine levels as prognostic parameter for overall survival and disease specific survival. Serum creatine values were dichotomized at the cut off value of $1.2 \mathrm{mg} / \mathrm{dl}$, since serum creatinine levels above $1.2 \mathrm{mg} / \mathrm{dl}$ are defined pathological. Variables that were significant prognostic parameters in univariate analysis were included into multivariate analyses, except for nodal status, which is an intrinsic part of FIGO stage. p-Values of $\leq 0.01$ and $\leq 0.05$ for univariate and multivariate analysis respectively were considered statistically significant. Hazard ratios (HR) and $95 \%$ confidence intervals (CI) are provided. Statistical analysis was performed using the Statistical Package for the Social Sciences statistical software (SPSS 24.0 for MAC, IBMCorp., Armonk, NY, USA). All data are available as Supplementary Database 1.

\title{
Results
}

The data of 341 patients with vulvar cancer was available. A total of 171 patients had to be excluded from analysis because of either non-invasive vulvar cancer or incomplete clinical or laboratory data. In total, the data of 170 patients with invasive vulvar cancer (FIGO Ib-FIGO IVb) and complete clinical information was included in the analysis. Table 1 shows patient characteristics. In general, the mean serum creatinine level (SD) of patients with vulvar cancer was $0.91 \pm 0.28 \mathrm{mg} / \mathrm{dl}$. Pathologically elevated serum creatinine levels $(\geq 1.20 \mathrm{mg} / \mathrm{dl})$ were measured in 17 patients $(10.0 \%)$. Table 2 shows the correlation of serum creatinine levels with clinicopathologic findings. Serum creatinine levels had no effect on patients' treatment (Table S2 of the supplemental material). Elevated serum creatinine levels were associated with patient's age $(\mathrm{p}<0.001)$, patient's performance status $(\mathrm{p}<0.001)$ and histologic grading $(\mathrm{p}=0.02)$. 


\begin{tabular}{|c|c|}
\hline Parameter & $\mathrm{N}(\%)$ or mean $(\mathrm{SD})$ \\
\hline No. of patients & 170 \\
\hline Mean creatinine value & $0.91(0.28)$ \\
\hline Patients' age & $67.7(14.0)$ \\
\hline \multicolumn{2}{|l|}{ FIGO Tumor stage } \\
\hline FIGO I & $95(55.9)$ \\
\hline FIGO II & $30(17.6)$ \\
\hline FIGO III & $32(18.8)$ \\
\hline FIGO IV & $13(7.6)$ \\
\hline \multicolumn{2}{|l|}{ Histologic Grading } \\
\hline G1 & $42(24.7)$ \\
\hline G2 & $97(57.1)$ \\
\hline G3 & $29(17.1)$ \\
\hline unknown & $2(1.2)$ \\
\hline \multicolumn{2}{|l|}{ Nodal involvement } \\
\hline N0 & $90(52.9)$ \\
\hline $\mathrm{N} 1$ & $56(32.9)$ \\
\hline Not assessed & $20(11.8)$ \\
\hline unknown & $4(2.4)$ \\
\hline \multicolumn{2}{|l|}{ ECOG status } \\
\hline 0 & $92(54.1)$ \\
\hline 1 & $48(28.2)$ \\
\hline 2 & $13(7.6)$ \\
\hline 3 & $5(2.9)$ \\
\hline unknown & $12(7.1)$ \\
\hline BMI & $28.0(6.9)$ \\
\hline \multicolumn{2}{|l|}{ Metastatic disease } \\
\hline M0 & $163(95.9)$ \\
\hline M1 & $7(4.1)$ \\
\hline Time of follow-up (months) & $26.5(\mathrm{IQR} 10-62)^{*}$ \\
\hline \multicolumn{2}{|l|}{ Status at last observation } \\
\hline Alive with no evidence of disease & $94(55.3)$ \\
\hline Alive with stable disease & $4(2.4)$ \\
\hline Progression & $13(7.6)$ \\
\hline Deceased & $59(34.7)$ \\
\hline
\end{tabular}

Table 1. Study patient characteristics. *Given as median and interquartile range. FIGO International Federation of Gynecologists and Obstetrics, ECOG European cooperative oncology group.

The median overall survival and disease free survival rates were 26.5 (IQR 10-62) months and 17.0 (IQR 5-53) months, respectively. In univariate analysis serum creatinine levels were evaluated as a continuous variable as well as a dichotomous variable using a cut-off of $1.2 \mathrm{mg} / \mathrm{dl}$. The 3 -year overall survival rate for patients with a serum creatinine level $\leq 1.2 \mathrm{mg} / \mathrm{dl}$ was $74.8 \%$ compared to $32.5 \%$ in patients with a serum creatinine level above $1.2 \mathrm{mg} / \mathrm{dl}(\mathrm{p}<0.001)$. In addition to FIGO tumor stage, patient's age, nodal involvement, and serum creatinine levels considered as continuous variable and dichotomous variable were significantly associated with poor overall survival (Table 3 ).

The overall survival and the disease specific survival of patients dependent on the serum creatinine level is shown in Figs 1 and 2, respectively. A multivariate analysis model revealed serum creatinine levels, FIGO tumor stage and patient's age to be independently associated with overall survival in patients with vulvar cancer. Table 4 shows univariate and multivariate survival analysis for the endpoint of disease specific survival.

\section{Discussion}

The present study investigated the prognostic value of pretherapeutic serum creatinine levels in patients with vulvar cancer. As a significant association between elevated pretherapeutic serum creatinine levels and poor overall survival in patients with invasive vulvar cancer, independent of established prognostic factors, was observed, several additional analysis were performed. Survival analyses for the endpoint of disease free survival were performed to support the finding of reduced overall survival. To elucidate the underlying mechanisms the relationship between serum creatinine levels and established risk factors were correlationally analysed.

The underlying mechanism of the association between elevated serum creatinine levels and diminished survival in patients with malignant diseases is subject to ongoing discussion. Several hypotheses have been proposed. 


\begin{tabular}{|c|c|c|c|}
\hline Parameter & $\begin{array}{l}\text { Mean } \\
\text { Creatinine (mg/ } \\
\text { dl) }\end{array}$ & p-value & $\begin{array}{l}\text { Missing } \\
\text { values }\end{array}$ \\
\hline Age & & $<0.001^{\mathrm{a}}$ & 0 \\
\hline$\leq 67.7$ & $0.79(0.16)$ & & \\
\hline$>67.7$ & $0.99(0.33)$ & & \\
\hline Tumor stage & & $0.49^{\mathrm{b}}$ & 0 \\
\hline FIGO I & $0.90(0.28)$ & & \\
\hline FIGO II & $0.97(0.34)$ & & \\
\hline FIGO III & $0.87(0.20)$ & & \\
\hline FIGO IV & $0.86(0.36)$ & & \\
\hline Grading & & $0.02^{\mathrm{a}}$ & 2 \\
\hline G1 & $0.73(0.44)$ & & \\
\hline G2-3 & $0.88(0.32)$ & & \\
\hline Nodal involvement & & $0.44^{\mathrm{a}}$ & 4 \\
\hline No & $0.89(0.25)$ & & \\
\hline $\mathrm{N} 1$ & $0.93(0.30)$ & & \\
\hline \multicolumn{4}{|l|}{ Performance Status } \\
\hline 0 & $0.88(0.22)$ & $<0.001^{\mathrm{b}}$ & 12 \\
\hline 1 & $0.86(0.22)$ & & \\
\hline 2 & $1.15(0.43)$ & & \\
\hline 3 & $1.35(0.77)$ & & \\
\hline
\end{tabular}

Table 2. Mean (SD) pre-treatment serum creatinine levels in patients with vulva cancer categorized by clinicopathologic findings. a students' t-test, ${ }^{\text {} O n e-w a y ~ A n o v a, ~ F I G O ~ I n t e r n a t i o n a l ~ F e d e r a t i o n ~ o f ~ G y n e c o l o g i s t s ~ a n d ~}$ Obstetrics, ECOG European cooperative oncology group.

\begin{tabular}{|l|l|l|l|l|l|l|}
\hline \multirow{2}{*}{ Parameter } & \multicolumn{2}{|l|}{ univariate } & \multicolumn{2}{l|}{ multivariate } \\
\hline & 3 -ys OS & $\mathbf{p}$-value & Comparison & HR & $\mathbf{9 5 \%}$-CI & p-value \\
\hline Serum Creatinine & & $<0.001$ & & 2.6 & $1.3-5.3$ & 0.006 \\
\hline$\leq 1.20$ & $74.8 \%$ & & & & & \\
\hline$>1.20$ & $32.5 \%$ & & & & & \\
\hline Serum Creatinine & & 0.01 & & & & \\
\hline FIGO tumor stage & & $<0.001$ & I vs. II vs. III vs. IV & 1.8 & $1.40-2.30$ & $<0.001$ \\
\hline FIGO I & $82.1 \%$ & & & & & \\
\hline FIGO II & $59.9 \%$ & & & & & \\
\hline FIGO III & $50.4 \%$ & & & & & \\
\hline FIGO IV & $52.7 \%$ & & & & & \\
\hline Age & & $<0.001$ & >vs. $\leq 66.5$ years & 2.9 & $1.6-5.2$ & $<0.001$ \\
\hline$\leq 67.7$ & $82.3 \%$ & & & & & \\
\hline$>67.7$ & $59.9 \%$ & & & & & \\
\hline Grading & & 0.64 & & & & \\
\hline G1 & $78.2 \%$ & & & & & \\
\hline G2/G3 & $67.5 \%$ & & & & & \\
\hline Nodal involvement & & $<0.001$ & & & & \\
\hline N0 & $81.7 \%$ & & & & & \\
\hline N1 & $51.7 \%$ & & & & & \\
\hline
\end{tabular}

Table 3. Univariate and multivariate overall survival analysis in 170 patients with invasive vulvar cancer. FIGO International Federation of Gynecologists and Obstetrics, OS disease specific survival, HR hazard ratio, CI confidence interval. Nodal involvement was not included into the multivariate model, since the nodal status is a crucial part of FIGO stage.

Firstly, elevated serum creatinine levels reflect impaired kidney function. Impaired kidney function is particularly known to increase cardiovascular mortality ${ }^{15}$. However, recent evidence showed that impaired kidney function is not only associated with cardiovascular mortality but also with non- cardiovascular mortality and with poor prognosis in different cancer patients ${ }^{15,16}$. Interestingly, the current study revealed that elevated serum creatinine levels were also associated with poor overall survival in patients with vulvar cancer. In addition, the disease specific survival of patients was also diminished in patients with elevated serum creatinine levels. This analysis was performed to corroborate the finding that elevated serum creatine levels are associated to poor 


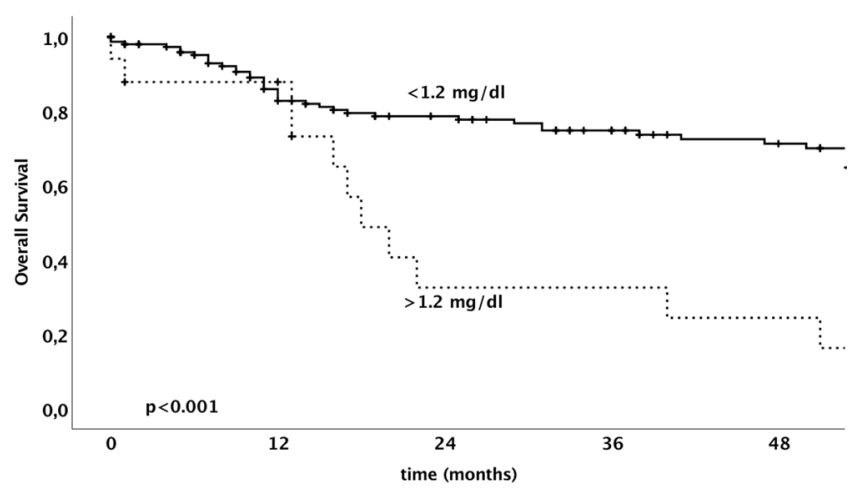

Figure 1. Kaplan-Meier analysis of overall survival of patients with vulvar cancer dependent on serum creatinine level using a cut-off of $1.2 \mathrm{mg} / \mathrm{dI}$.

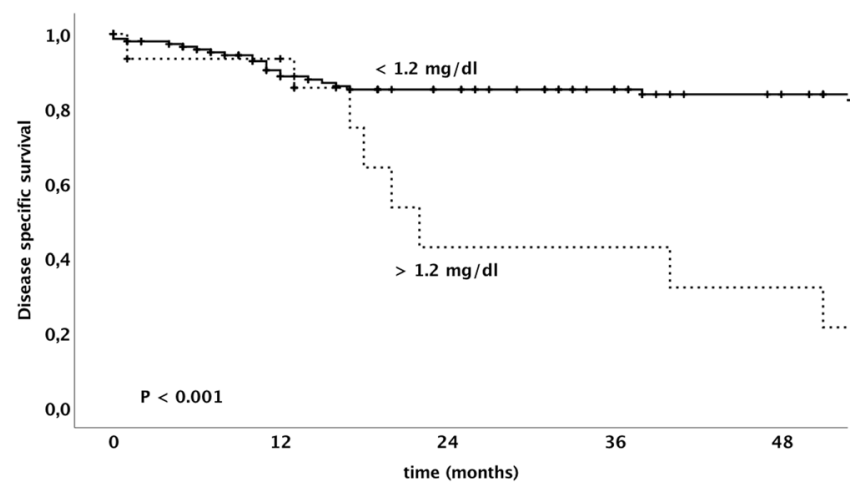

Figure 2. Kaplan-Meier analysis of disease specific of patients with vulvar cancer dependent on serum creatinine level using a cut-off of $1.2 \mathrm{mg} / \mathrm{dI}$.

\begin{tabular}{|l|l|l|l|l|l|l|}
\hline \multirow{2}{*}{ Parameter } & \multicolumn{2}{|l|}{ univariate } & \multicolumn{2}{l|}{ multivariate } \\
\hline & $\mathbf{3}$-ys DSS & p-value & Comparison & HR & $\mathbf{9 5 \% - C I}$ & p-value \\
\hline Serum Creatinine & & $<0.001$ & >vs. $\leq 1.2 \mathrm{mg} / \mathrm{dl}$ & 3.8 & $1.6-9.0$ & 0.002 \\
\hline$\leq 1.20$ & $85.1 \%$ & & & & & \\
\hline$>1.20$ & $42.8 \%$ & & & & & \\
\hline Serum Creatinine & & 0.005 & & & & \\
\hline FIGO tumor stage & & $<0.001$ & I vs. II vs. III vs. IV & 2.0 & $1.5-2.8$ & $<0.001$ \\
\hline FIGO I & $93.6 \%$ & & & & & \\
\hline FIGO II & $59.9 \%$ & & & & & \\
\hline FIGO III & $63.1 \%$ & & & & & \\
\hline FIGO IV & $72.5 \%$ & & & & & \\
\hline Age & & 0.014 & >vs. $\leq 67.7$ years & 2.3 & $1.0-5.0$ & 0.038 \\
\hline$\leq 67.7$ & $86.7 \%$ & & & & & \\
\hline$>67.7$ & $76.5 \%$ & & & & & \\
\hline Grading & & 0.07 & & & & \\
\hline G1 & $89.2 \%$ & & & & & \\
\hline G2/3 & $78.6 \%$ & & & & & \\
\hline Nodal involvement & & $<0.001$ & & & & \\
\hline N0 & $92.1 \%$ & & & & & \\
\hline N1 & $60.7 \%$ & & & & & \\
\hline
\end{tabular}

Table 4. Univariate and multivariate disease specific survival analysis in 170 patients with invasive vulvar cancer. FIGO International Federation of Gynecologists and Obstetrics, DSS disease specific survival, HR hazard ratio, CI confidence interval. Nodal involvement was not included into the multivariate model, since the nodal status is a crucial part of FIGO stage. 
overall survival. The observed association might indicate that this is not simply a side effect of the fact that patients with impaired kidney function have in general a decreased prognosis but may be potentially directly related to the malignant disease.

The creatine/creatinine metabolism not only plays a major role in energy production of muscle cells but has also been linked to carcinogenesis and cancer progression. Creatine and Phosphocreatine are essential energy sources donating ATP. Creatinine is a waste product of the creatine metabolism ${ }^{17}$. Preclinical studies showed during cancer progression creatine synthesizing enzymes to be significantly upregulated, while creatine levels are constantly decreasing ${ }^{18}$. Thus, it can be speculated that creatine is quickly utilized by the cancer cell as an energy resource, which may lead to creatinine as a waste product. Hence, it seems plausible, that elevated serum creatinine levels are associated with highly active tumors. In the current study, high creatinine levels were associated with grade 2 and 3 cancer. In addition, it seems therefore plausible that elevated serum creatinine levels are also associated with shortened disease specific survival in this study.

In contrast, serum creatinine levels were not associated with tumor stage. Thus, elevated serum creatinine levels were not simply caused by large tumor masses leading to urinary tract obstruction.

Secondly, evidence suggests that chronic low-grade inflammation may induce intra-renal vascular dysfunction that can lead to elevated serum creatinine levels ${ }^{10}$. Links between inflammation and cancer have been established for decades, and it has been shown that the tumor environment may sustain circle of tumor progression and inflammatory response ${ }^{19,20}$. Hence, elevated serum creatinine levels as seen in this study may mirror a chronic inflammatory reaction, triggered by the underlying malignancy.

In either case, if serum creatinine levels are increased in patients, a diagnostic algorithm as suggested by current guidelines should be applied to elucidate the underlying source of provenience ${ }^{21}$.

To our knowledge this is the first study to describe pretherapeutic elevated serum creatinine levels as an independent prognostic parameter for overall survival in patients with vulvar cancer. Undoubtedly, this study suffered from limitations due to its retrospective design. The treatment of patients with vulvar cancer changed and survival increased significantly over the last two decades. This might have had an impact on the results of the current study. On the other hand, we were able to include a relatively large homogenous cohort of 170 patients with vulvar cancer that was followed-up for a long period of time at a single institution.

Finally, we present pretherapeutic serum creatinine levels as a novel independent prognostic parameter in patients with vulvar cancer. This novel biomarker may be used for individual risk estimation, patient counseling and may be integrated in more advanced prognostic models for vulvar cancer.

In addition, it would be interesting to confirm our findings in a prospective study design and investigate whether pretherapeutic correction of high creatinine levels improves the survival in patients with vulvar cancer.

\section{References}

1. National Cancer Institute, S. Epidemiology, and End Results Program. Cancer Stat Facts: Vulvar Cancer, https://seer.cancer.gov/ statfacts/html/vulva.html (2018).

2. Judson, P. L., Habermann, E. B., Baxter, N. N., Durham, S. B. \& Virnig, B. A. Trends in the incidence of invasive and in situ vulvar carcinoma. Obstetrics and gynecology 107, 1018-1022, https://doi.org/10.1097/01.AOG.0000210268.57527.a1 (2006).

3. Woelber, L. et al. Clinicopathological prognostic factors and patterns of recurrence in vulvar cancer. Anticancer research 29, 545-552 (2009).

4. Woelber, L. et al. Prognostic role of lymph node metastases in vulvar cancer and implications for adjuvant treatment. Int J Gynecol Cancer 22, 503-508, https://doi.org/10.1097/IGC.0b013e31823eed4c (2012).

5. Gadducci, A., Cionini, L., Romanini, A., Fanucchi, A. \& Genazzani, A. R. Old and new perspectives in the management of high-risk, locally advanced or recurrent, and metastatic vulvar cancer. Crit Rev Oncol Hematol 60, 227-241, https://doi.org/10.1016/j. critrevonc.2006.06.009 (2006).

6. Polterauer, S. et al. Prognostic value of lymph node ratio and number of positive inguinal nodes in patients with vulvar cancer. Gynecol Oncol 147, 92-97, https://doi.org/10.1016/j.ygyno.2017.07.142 (2017).

7. Woelber, L. et al. Role of tumour-free margin distance for loco-regional control in vulvar cancer-a subset analysis of the Arbeitsgemeinschaft Gynakologische Onkologie CaRE-1 multicenter study. European journal of cancer 69, 180-188, https://doi. org/10.1016/j.ejca.2016.09.038 (2016).

8. Hefler-Frischmuth, K. et al. The inflammation-based modified Glasgow Prognostic Score in patients with vulvar cancer. Eur J Obstet Gynecol Reprod Biol 149, 102-105, https://doi.org/10.1016/j.ejogrb.2009.12.027 (2010).

9. Seebacher, V. et al. The impact of plasma fibrinogen levels on patients with vulvar cancer. Eur J Obstet Gynecol Reprod Biol 161, 88-91, https://doi.org/10.1016/j.ejogrb.2011.11.030 (2012).

10. Panotopoulos, J. et al. Elevated serum creatinine and low albumin are associated with poor outcomes in patients with liposarcoma. J Orthop Res 34, 533-538, https://doi.org/10.1002/jor.23002 (2016).

11. Morizane, S. et al. Preoperative prognostic factors after radical nephroureterectomy in patients with upper urinary tract urothelial carcinoma. Int Urol Nephrol 45, 99-106, https://doi.org/10.1007/s11255-012-0347-1 (2013).

12. Rausch, S. et al. Impaired estimated glomerular filtration rate is a significant predictor for non-muscle-invasive bladder cancer recurrence and progression-introducing a novel prognostic model for bladder cancer recurrence. Urol Oncol 32, 1178-1183, https:// doi.org/10.1016/j.urolonc.2014.05.009 (2014).

13. Aapro, M. \& Launay-Vacher, V. Importance of monitoring renal function in patients with cancer. Cancer Treat Rev 38, 235-240, https://doi.org/10.1016/j.ctrv.2011.05.001 (2012).

14. Pecorelli, S. Revised FIGO staging for carcinoma of the vulva, cervix, and endometrium. International journal of gynaecology and obstetrics: the official organ of the International Federation of Gynaecology and Obstetrics 105, 103-104 (2009).

15. Fried, L. F. et al. Renal insufficiency as a predictor of cardiovascular outcomes and mortality in elderly individuals. J Am Coll Cardiol 41, 1364-1372 (2003).

16. Fried, L. F. et al. Kidney function as a predictor of noncardiovascular mortality. J Am Soc Nephrol 16, 3728-3735, https://doi. org/10.1681/ASN.2005040384 (2005).

17. Wyss, M. \& Kaddurah-Daouk, R. Creatine and creatinine metabolism. Physiol Rev 80, 1107-1213, https://doi.org/10.1152/ physrev.2000.80.3.1107 (2000).

18. Patra, S. et al. A short review on creatine-creatine kinase system in relation to cancer and some experimental results on creatine as adjuvant in cancer therapy. Amino Acids 42, 2319-2330, https://doi.org/10.1007/s00726-011-0974-3 (2012).

19. Schwameis, R. et al. The Prognostic Value of C-Reactive Protein Serum Levels in Patients with Uterine Leiomyosarcoma. PloS one 10, e0133838, https://doi.org/10.1371/journal.pone.0133838 (2015). 
20. Balkwill, F. Cancer and the chemokine network. Nat Rev Cancer 4, 540-550, https://doi.org/10.1038/nrc1388 (2004).

21. NICE. Chronic kidney disease in adults: assessment and management, https://www.nice.org.uk/guidance/cg182/resources/chronickidney-disease-in-adults-assessment-and-management-pdf-35109809343205 (2014).

\section{Acknowledgements}

This study was sponsored by the Department of General Gynecology and Gynecologic Oncology, Medical University Vienna and the Karl Landsteiner Institute for General Gynecology and Experimental Gynecologic Oncology, Vienna, Austria.

\section{Author Contributions}

R.S. conceived the manuscript, performed data acquisition, analyzed data, M.P. performed data acquisition, C.B. performed data acquisition, wrote ethics protocol, L.H. performed data acquisition, A.R. conceived manuscript, performed data analysis, V.S. performed data acquisition, G.C. conceived the manuscript, performed data acquisition, analyzed data, S.P. performed data acquisition, performed data analysis, H.S. performed additional statistical analysis, revised manuscript All authors have reviewed the final version of the manuscript.

\section{Additional Information}

Supplementary information accompanies this paper at https://doi.org/10.1038/s41598-019-47560-3.

Competing Interests: The authors declare no competing interests.

Publisher's note: Springer Nature remains neutral with regard to jurisdictional claims in published maps and institutional affiliations.

(1) Open Access This article is licensed under a Creative Commons Attribution 4.0 International License, which permits use, sharing, adaptation, distribution and reproduction in any medium or format, as long as you give appropriate credit to the original author(s) and the source, provide a link to the Creative Commons license, and indicate if changes were made. The images or other third party material in this article are included in the article's Creative Commons license, unless indicated otherwise in a credit line to the material. If material is not included in the article's Creative Commons license and your intended use is not permitted by statutory regulation or exceeds the permitted use, you will need to obtain permission directly from the copyright holder. To view a copy of this license, visit http://creativecommons.org/licenses/by/4.0/.

(C) The Author(s) 2019 